\title{
Correlation between shrinkage and infection of implanted synthetic meshes using an animal model of mesh infection
}

\author{
Laurent Mamy $\cdot$ Vincent Letouzey \\ Jean-Philippe Lavigne • Xavier Garric • Jean Gondry • \\ Pierre Mares $\cdot$ Renaud de Tayrac
}

Received: 2 April 2010 / Accepted: 1 August 2010

(C) The International Urogynecological Association 2010

\begin{abstract}
Introduction and hypothesis The aim of this study was to evaluate a link between mesh infection and shrinkage. Methods Twenty-eight Wistar rats were implanted with synthetic meshes that were either non-absorbable (polypropylene (PP), $n=14$ ) or absorbable (poly (D,L-lactic acid) (PLA94), $n=14)$. A validated animal incisionnal abdominal hernia model of mesh infection was used. Fourteen meshes ( $n=7$ PLA94 and $n=7$ PP meshes) were infected intraoperatively with $10 \mathrm{e} 6 \mathrm{CFU}$ Escherichia coli, and compared with 14 non-infected meshes ( $n=7$ PLA94 and $n=7$ PP meshes) (control groups). Explantations were performed on day 30. Shrinkage was evaluated by a reproducible numerical analysis of mesh area. Infection and histological study were evaluated on day 30 .
\end{abstract}

L. Mamy $\cdot$ V. Letouzey $\cdot$ P. Mares $\cdot$ R. de Tayrac

Department of Obstetrics and Gynecology, Carémeau Hospital,

Nîmes, France

L. Mamy $\cdot$ J. Gondry

Department of Obstetrics and Gynecology, Centre of Gynecology and Obstetrics of Amiens,

Amiens, France

J.-P. Lavigne

INSERM, Espri 26, Universite Montpellier 1, UFR Medecine,

Nîmes, France

\section{Garric}

Faculty of Pharmacy, IBMM, UMR CNRS 5473,

University of Montpellier 1,

Montpellier, France

\section{R. de Tayrac $(\bowtie)$}

Service de Gynécologie-Obstétrique, Hôpital Carémeau,

Place du Pr Robert Debré,

30029 Nîmes Cedex 9, France

e-mail: renaud.detayrac@chu-nimes.fr
Results Non-infected meshes were less shrunk than infected meshes for both non-absorbable $(5.0 \pm 1.7 \%$ versus $21.6 \pm 6.1 \%, p<0.05)$ and absorbable meshes $(2.4 \pm 0.9 \%$ versus $11.0 \pm 2.5 \%, p<0.05$ ).

Conclusion This study highlights a link between infection and shrinkage in the model used.

Keywords Infection · Mesh · Poly (lactic acid) .

Polypropylene $\cdot$ Shrinkage

\section{Introduction}

The surgical treatment of genital prolapse using synthetic mesh is restricted by complications such as erosion, infection and shrinkage. Vaginal erosions are well known and may affect more than $10 \%$ of patients [1]. They usually require local excision. However, recent publications have reported lower erosion rates $(0 \%$ to $6.9 \%)$, which are therefore close to those observed after laparoscopic or open sacrocolpopexy ( $3 \%$ to $5 \%$ ) [2].

The biological response of tissues to foreign bodies largely depends on the material and conformation of the mesh [3]. The current literature supports the notion that monofilament polypropylene with a large-pore size induces fewer complications [4]. However, data are not consistently compelling, making it difficult to choose the ideal material [5]. Furthermore, infection is thought to be responsible for erosion, shrinkage, migration of prosthetic devices, and pain [6]. Microorganisms can interfere with the integration process through adhesion to mesh surfaces, and such adhesion is an important stage in the infection [7]. The first stage of adhesion is physical and reversible. The second stage is molecular and irreversible [8]. A subclinical mesh infection, acquired during the initial implantation, 
could result in wound separation with subsequent mesh exposure [9]. Both infection and shrinkage are current problems concerning the treatment of genital prolapse using synthetic meshes.

The aim of this study was to evaluate the link between mesh infection and shrinkage in a validated animal model of mesh infection.

\section{Materials and methods}

Shrinkage was studied in relation to the infected or noninfected character of the meshes used. A referent mesh, light-weight PP (Parietene ${ }^{\circledR}$, Sofradim-Covidien, Trévoux, France), weighting $38 \mathrm{~g} / \mathrm{m}^{2}$, was compared with a new

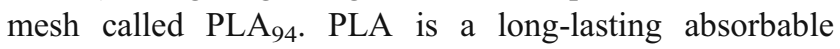
polymer that has been used for many years in not only orthopedic and vascular surgery, due to its biocompatibility, but also in preclinic study of our team $[10,11]$. We used a thread composed of PLA with 94\% L-lactic acid and 6\% Dlactic acid $\left(\mathrm{PLA}_{94}\right)$, which had previously been treated by extraction to remove the surface additive and any agents present in the fibers. A large-pore monofilament mesh composed only of $\mathrm{PLA}_{94}$ was used. Time to resorption depends on the level of "L" form containing in the polymer. The resorption time of $\mathrm{PLA}_{94}$ is done in 1.5 years; nonetheless, after 8 months, the mesh has no mechanical property anymore. The technical characteristics of the two meshes are outlined in Table 1. Meshes were pre-cut into $30 \times 30-\mathrm{mm}$ patches. Eight 3/0 polypropylene sutures were placed around each implant to facilitate surface area measurements. All meshes used for the study were then sterilized by ethylene oxide, which does not affect their mechanical properties [11].

Ethical committee approval was obtained for the animal study (Ethical Committee Approval number CE-LR-0804).

Rats were housed in an animal facility and treated in accordance with current national guidelines on animal welfare. Twenty-eight Wistar rats (weight range 250 to $300 \mathrm{~g}$ ) were implanted with synthetic meshes that were either non-absorbable (polypropylene (PP), $n=14$ ) or absorbable (poly (D,L-lactic acid) $\left.\left(\mathrm{PLA}_{94}\right), n=14\right)$. Fourteen meshes ( $n=7$ PLA $_{94}$ meshes and $n=7$ PP meshes) were infected intraoperatively with 10e6 CFU Escherichia coli and compared with 14 non-infected meshes ( $n=7$ PLA $_{94}$ meshes and $n=7$ PP meshes) (control groups).
The animals were anesthetized by a mixture of halotane $2.5 \%$ and oxygen $(0.5 \mathrm{l} / \mathrm{min})$ administered via an inhalation mask, and by an intraperitoneal injection of ketamine $(50 \mathrm{mg} / \mathrm{kg})$. The experiments were performed according to the validated incisional hernia rat model described by Alponat et al. [12] and used by Zheng et al. [13]. The abdomen was shaved, disinfected with betadine, and draped to ensure sterile conditions. A vertical midline incision was made through the skin, and skin flaps were raised. A $15 \times 25-\mathrm{mm}$ longitudinal full-thickness defect was created. The defects were repaired with the meshes positioned under the skin but above fascia and muscles. Typically, a mesh was extended $5 \mathrm{~mm}$ beyond the cranial and caudal borders and $15 \mathrm{~mm}$ laterally. The mesh was laid over the defect with a slight overlap and was sutured without tension to the abdominal wall using interrupted resorbable 2/0 PGA sutures (Vicryl ${ }^{\circledR}$, Ethicon) at the fourth corners. The peritoneum was left unclosed under the mesh. The meshes were then photographed in vivo using a digital reflex camera (Canon EOS 400D digital), and the area of the mesh was evaluated numerically by ImageJ freeware available on the internet (results in pixels) (Fig. 1). The distance between the camera and the mesh was noted such that the photograph at explantation on day 30 was taken with the same distance between mesh and camera (average $40 \mathrm{~cm}$ ). The subcutaneous tissues and skin incision were closed with interrupted resorbable $2 / 0$ PGA sutures $\left(\right.$ Vicryl $\left.^{\circledR}\right)$. Bacterial inoculation was performed at the end of the surgical procedure according to a validated model of mesh infection previously developed by our team [14], using a small quantity of $E$. coli (10e6 CFU) in order to mimic a subclinical mesh infection.

The animals were checked daily for local or systemic complications throughout the entire 30-day observation period. Explantations were performed on day 30. The meshes were photographed in vivo using the same reflex digital camera previously used, and mesh area was again evaluated numerically by the software (Fig. 2). The initial implants, with their neighboring host tissues, were resected. On retrieval, the meshes were cut into two strips for both histological and bacteriological study.

An uropathogenic E. coli (UPEC) strain previously isolated from a patient with UTI (NECS19923) and harboring the main virulence factors (e.g., toxins, adhesins, siderophores, and capsules) registered in UPEC was used.

Table 1 Comparative technical characteristics of the implanted meshes

\begin{tabular}{llllllll}
\hline Name & Type of textile & Filament & Structure & Coating & Pore size $(\mathrm{mm})$ & Amid classification & Weight $\left(\mathrm{g} / \mathrm{m}^{2}\right)$ \\
\hline None & PLA $_{94}$ & Mono & Knitted & No & Macroporous $(3.5 \times 1.7)$ & I & 100 \\
Parietene light & PP light & Mono & Knitted & No & Macroporous $(1.5 \times 1.7)$ & I & 38 \\
\hline
\end{tabular}


Fig. 1 Mesh contraction was evaluated by measuring mesh area pre- and postoperatively using a standardized protocol of in vivo mesh photography. Pictures of anesthetized rats at a distance of $40 \mathrm{~cm}$ were taken, before skin closure preoperatively and after skin incision on day 30
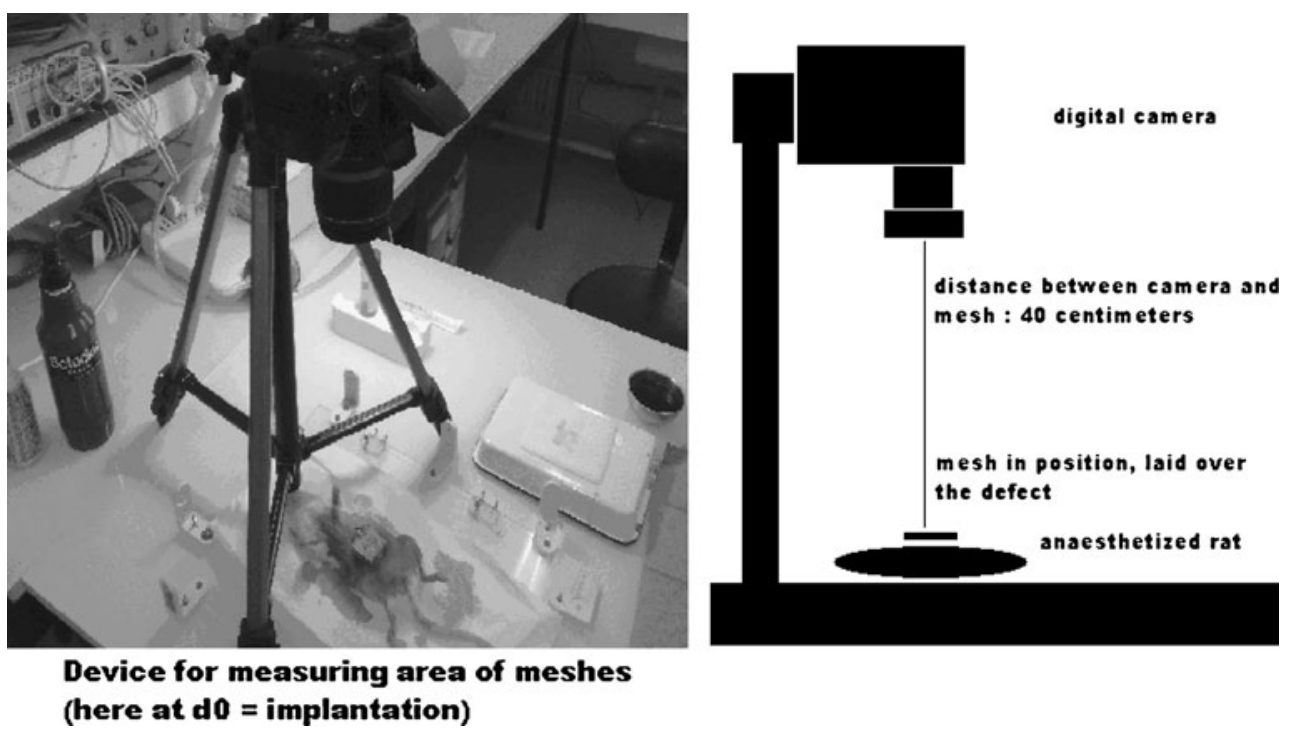

(here at do = implantation)
The bacteriologist was blinded with regard to mesh identity. Strips samples were placed in $2 \mathrm{ml}$ Muller-Hinton broth, crushed with a sterile scalpel, and incubated at $37^{\circ} \mathrm{C}$ for 18 h. Colonies were counted after serial dilution. Different standard agar plates were used to identify and isolate the bacteria that grew around the meshes. Genus and species were determined biochemically using the API identification card (bioMérieux, Marcy l'etoile, France). Post-infection $E$. coli strains were identified by genetically comparing randomly collected colonies with NECS19923 on the basis of their virulence profile.

The investigator for the histopathological examination was blinded to the mesh. For each histology strip sample (one per mesh explanted), three slides were stained with Masson's trichrome, and three slides with hematoxylin and eosin (H\&E). The microscopic evaluation quantified the presence of polynuclear, mononuclear, and giant cells; newly formed vessels in H\&E-stained sections; and collagen (organization, composition, and amount). Five fields per slide were counted at $\times 400$ magnification (Olympus AX70 Provis, Japan). The scale used was similar to that described by Zheng et al. [13].

Data were summarized by frequency and percentage for categorical variables and by mean, standard deviation, median, and range for continuous variables. To investigate the association between categorical variables, univariate statistical analyze were performed using Pearson's chi-2 test or Fisher's exact test if the sample size was small and using Wilcoxon's test or Student's $t$ test for continuous variables. In order to account for the number of tests performed, the alpha risk was corrected using the Bonferroni method. All statistical tests were two-tailed, and a $p$ value of less than 0.05 was considered statistically significant. All statistical tests were performed using SAS v.9 statistical software (SAS Institute, Cary, NC, USA).

\section{Results}

Four animals died during the experimental period (14.3\%): one in the $\mathrm{PLA}_{94}$-infected group, two in the PP-infected group, and one in the PP non-infected group. One died after anesthesia and three because of postoperative evisceration. None died because of infection. Tests conducted on day 30 showed that no $E$. coli were present on the mesh in noninfected animals. In the opposite group, the NECS19923 strain was found in all infected animals and there was at least 10e5 CFU. Contamination with Staphylococcus epidermidis was possible, but always less than 10e5 CFU, and did not correlate with mesh shrinkage.

Non-infected meshes were less contracted $(2.4 \pm 0.9 \%$ for $\mathrm{PLA}_{94}$ and $5.0 \pm 1.7 \%$ for PP) than infected meshes $(11.0 \pm$ $2.5 \%$ for $\mathrm{PLA}_{94}$ and $21.6 \pm 6.1 \%$ for PP) after 1 month of implantation $(p<0.05)$. Moreover, absorbable $\mathrm{PLA}_{94}$ meshes contracted less than non-absorbable PP meshes when infected $(11.0 \pm 2.5 \%$ versus $21.6 \pm 6.1, p<0.05)$ (Table 2). Histological results are shown in Table 3.

\section{Discussion}

Three recent randomized controlled trials have showed that mesh repair was superior to anterior colporrhaphy in pelvic floor reconstructive surgery [15-17]. Low-weight polypropylene meshes were used by Hiltunen et al. [15], and the rate of recurrence was better with it than with colporrhaphy repair. Nguyen and Burchette [16] also found less recurrence of prolapsed using polypropylene than without. Sivaslioglu et al. [17] concluded in their study that surgery with light polypropylene mesh (Sofradim, Parietene) is superior in terms of anatomical results to the site-specific surgery in the treatment of cystocoeles. Local complica- 
Fig. 2 Examples of mesh contraction measurements using Image J software

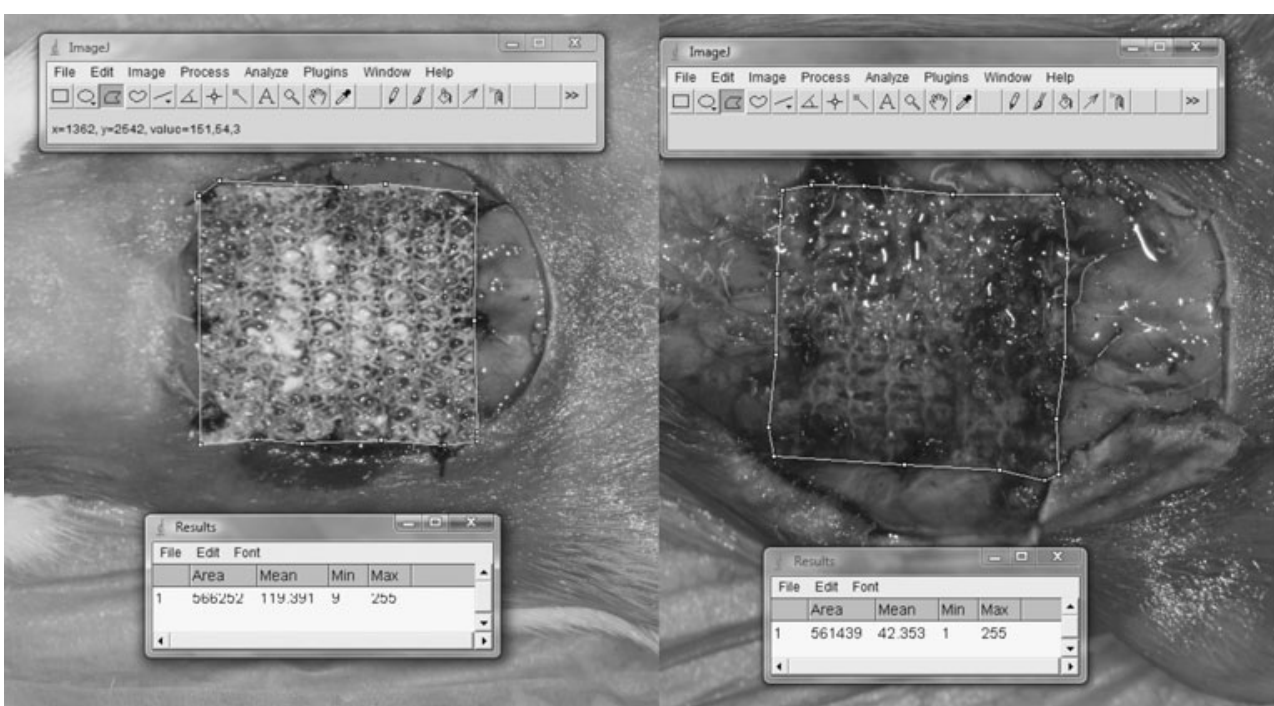

uninfected PLA94 mesh : contraction (measure in vivo) on the left at do, area $=\mathbf{5 6 6 2 5 2}$ pixels. on the right, at d30 area $=\mathbf{5 6 1 4 3 9}$ contraction : $(566252-561439) / 566252=0.8 \%$

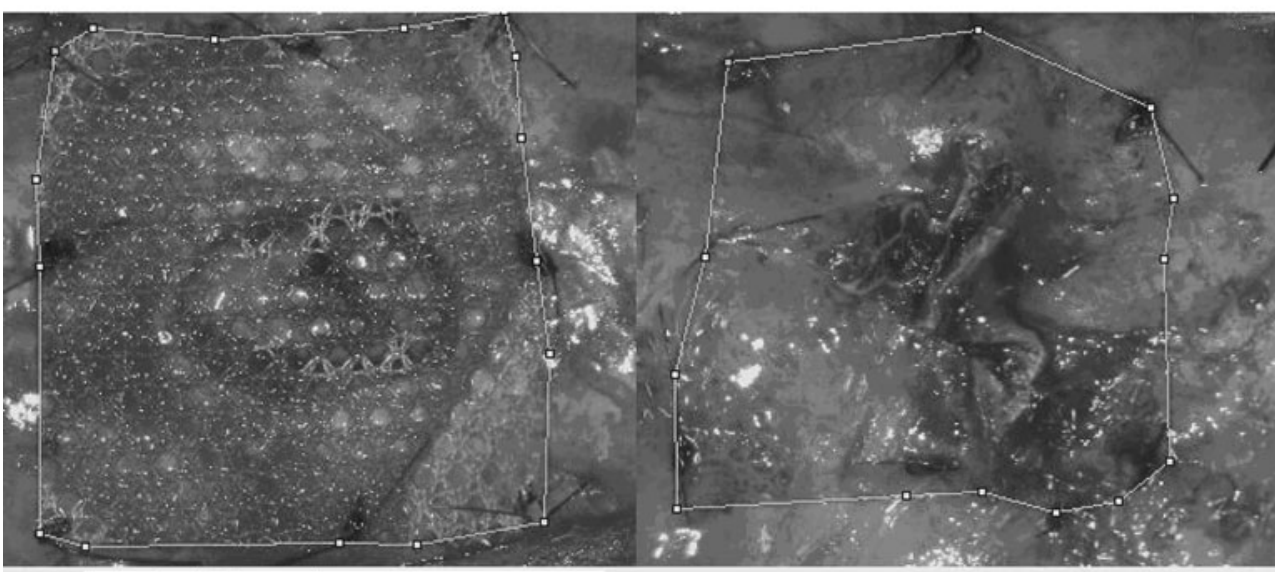

infected PP mesh mesh contraction (measurements in vivo) :

on the left, area of the mesh at day $0=632000$ pixels

on the right, area of the mesh at day $30=518342$ pixels contraction : $(632000-518342) / 632000=18 \%$ tions, such as erosion, shrinkage, pain, dyspareunia, and infection, are still important limitations, particularly in young and sexually active women.

Whereas the pathophysiology and management of vaginal erosion are now well known, little information is available on mesh shrinkage. Although this phenomenon is poorly defined, its incidence poorly reported, and its pathophysiology and risk factors unclear, it may be responsible for major local complications such as pain and dyspareunia, and also recurrences, with subsequent difficult reinterventions.

Hypotheses explaining mesh shrinkage include an inflammatory reaction around the mesh, itself correlated with weight and pore size, and an immunological reaction, which we have not found in our study, probably due to a lack of power. In the present study, we put forward the hypothesis that infection of the mesh caused by bacterial contamination during the implantation phase is an independent risk factor for shrinkage. When working with two different meshes, we observed a significant correlation between infection and shrinkage.

Tissue ingrowth around synthetic implants is a complex phenomenon, indissociable from the inflammatory reaction. An immunochemical analysis of infected implanted mesh would be of great interest, with a particular focus on TGF-betal which is a determinant of foreign body reaction to alloplastic materials in rat fibroblast cultures [18]. This type of study should be able to differentiate between the respective responsibilities of bacterial contamination and non-infectious foreign body reactions in mesh shrinkage. 
Table 2 Results for infection and contraction

\begin{tabular}{|c|c|c|c|c|c|}
\hline Meshes & Samples & Death & Infection on day30 (mean CFU) & Contraction $(\% \pm \mathrm{SD})$ & $p$ \\
\hline Non-infected PLA 94 & 7 & 0 & 0 & $2.4 \pm 0.9$ & - \\
\hline Infected $\mathrm{PLA}_{94}($ E. coli $10 \mathrm{e} 6 \mathrm{CFU})$ & 7 & 1 & $7(5 \times 10 \mathrm{e} 5)$ & $5 \pm 1.7$ & $<0.05^{\mathrm{a}}$ \\
\hline Non-infected PP & 7 & 1 & 0 & $11 \pm 2.5$ & - \\
\hline Infected PP (E. coli 10e6 CFU) & 7 & 2 & $7(2 \times 10 \mathrm{e} 6)$ & $21.6 \pm 6.1$ & $<0.05^{\mathrm{b}}$ \\
\hline
\end{tabular}

$P L A_{94}$ poly (lactic acid) mesh, $P P$ polypropylene mesh

${ }^{a}$ The difference between infected PLA meshes and non-infected PLA meshes is significant in term of shrinkage $(p<0.05)$ : The infected one are more shrunk

${ }^{\mathrm{b}}$ The difference between infected PPL meshes and non-infected PPL meshes is significant in term of shrinkage $(p<0.05)$ : The infected one are more shrunk

However, intraoperative bacterial contamination of synthetic implants is rather uncommon in clinical practice; although clinical infection such as abscess is rare, subclinical chronic infection may explain several local complications. This has already been observed in mesh exposure after wound separation due to infection [9]. Further studies are necessary to confirm our hypothesis using more animals, animal models for vaginal surgery, and longerterm experiments. Should the hypothesis be confirmed, the use of antibacterial products on synthetic implants would be greatly beneficial in women.

Mesh shrinkage has been reported to occur early after tissue implantation, within the first 14 days of tissue healing in response to the acute inflammatory reaction [19]. After this early phase, mesh shrinkage defined as surface loss measured in millimeters and expressed as a percentage of initial surface area, and which occurs in $72 \%$ of all implants, was stable over a 180 -day period in a rabbit abdominal model where it correspond to $3-20 \%$ of the initial surface area [19].

E. coli isolate was used because this strain is common in vaginal infections [20] and is the most frequent in infected biomaterial [21]. Prosthesis infection often springs from the transformation of a usually non-pathogenic colonizing bacterium into virulent colonies that adhere to the material [21]. A low infection level (10e6 UFC) was injected intraoperatively on the mesh in order to mimic a subclinical bacterial contamination of the mesh.

As previously shown [10], mesh was better tolerated in the $\mathrm{PLA}_{94}$ than in the PPL groups, and PLA 94 is also less likely to be infected than PPL, which could contribute to improving biocompatibility, but our results are not significant probably due to a lack of power. Anyway, PLA94 did shrink less than PPL. A better tolerance and a lower degree of infection with a better integration of the mesh could explain the difference; physical reasons may explain the difference, including different hydrophilic surface properties, which could influence bacteria colonization [22]. Furthermore, research with more power would be interesting in order to find out a link between shrinkage, infection, and integration of meshes.

In conclusion, shrinkage, which is a complex phenomenon occurring during tissue healing around synthetic biomaterial, may be significantly influenced by both the polymer used for the implant and intraoperative bacterial contamination around the mesh. To explain the mechanism of shrinkage with infection, more powerful studies are needed. Are the meshes less integrated when infected? Mechanical strength tests and histological studies with more effective power would be

Table 3 Histological results (scores from 0 to 3 )

\begin{tabular}{|c|c|c|c|c|c|c|c|}
\hline Meshes & $\begin{array}{l}\text { Mononuclear } \\
\text { cells }\end{array}$ & $\begin{array}{l}\text { Polynuclear } \\
\text { cells }\end{array}$ & $\begin{array}{l}\text { Foreign body } \\
\text { giant cells }\end{array}$ & Vascularity & $\begin{array}{l}\text { Collagen } \\
\text { organization }\end{array}$ & $\begin{array}{l}\text { Collagen } \\
\text { composition }\end{array}$ & $\begin{array}{l}\text { Collagen } \\
\text { amount }\end{array}$ \\
\hline $\mathrm{PLA}_{94}, n=7$ & $1.43^{\mathrm{a}}$ & 0.71 & 0.29 & 2.86 & 2.71 & 2.43 & 2.71 \\
\hline Infected PLA $_{94}, n=6$ & 2 & 0.83 & 0.33 & 2.5 & 2.5 & 2.67 & 2.8 \\
\hline PP, $n=6$ & 2.5 & 1.33 & 1 & 2.17 & 2 & 1.83 & 2 \\
\hline Infected PP, $n=5$ & 2.8 & 1.4 & 0.8 & 1.6 & 1.86 & 2 & 2 \\
\hline$p^{\mathrm{b}}$ & 0.43 & 1.0 & 1.0 & 0.18 & 0.84 & 0.68 & 1.0 \\
\hline
\end{tabular}

$P L A_{94}$ poly (lactic acid) mesh, $P P$ polypropylene mesh

${ }^{\text {a }}$ Results: average score of the parameters

${ }^{\mathrm{b}}$ Statistical comparison between the 14 non-infected meshes and the 14 infected meshes 
interesting. These observations are critical if the local complications of synthetic mesh placement by the vaginal route are to be reduced.

Conflicts of interest This study represents a master thesis funded by Covidien. Renaud de Tayrac is a consultant for Covidien. The other co-authors have no conflict of interest.

\section{References}

1. Collinet P, Belot F, Debodinance P, Ha Duc E, Lucot JP, Cosson M (2006) Transvaginal mesh technique for pelvic organ prolapse repair: mesh exposure management and risk factors. Int Urogynecol J Pelvic Floor Dysfunct 17:315-320

2. De Ridder D (2008) Should we use meshes in the management of vaginal prolapse? Curr Opin Urol 18:377-382

3. Bellon JM, Bujan J, Contreras L, Hernando A (1995) Integration of biomaterials implanted into abdominal wall: process of scar formation and macrophage response. Biomaterials 16:381-387

4. Birch C, Fynes MM (2002) The role of synthetic and biological prostheses in reconstructive pelvic floor surgery. Curr Opin Obstet Gynecol 14:527-535

5. Cosson M (2004) Risk of infection and prostheses: time out or a red flag? J Gynécol Obstét Biol Reprod 33:559-560

6. Dougherty SH (1988) Pathobiology of infection in prosthetic devices. Rev Infect Dis 10:1102-1117

7. An YH, Friedman RJ (1998) Concise review of mechanisms of bacterial adhesion to biomaterial surfaces. J Biomed Mater Res 43:338-348

8. Marshall K (1985) Mechanisms of bacterial adhesions at solid-water interfaces. In: Savage D, Fletcher M (eds) Bacterial adhesion. Mechanisms and physiological significance. Plenum, New York

9. Huang KH, Kung FT, Liang HM, Chang SY (2005) Management of polypropylene mesh erosion after intravaginal midurethral sling operation for female stress urinary incontinence. Int Urogynecol J Pelvic Floor Dysfunct 16:437-440

10. de Tayrac R, Oliva-Lauraire MC, Guiraud I, Henry L, Vert M, Mares P (2007) Long-lasting bioresorbable poly(lactic acid) 94 mesh: a new approach for soft tissue reinforcement based on an experimental pilot study. Int Urogynecol J Pelvic Floor Dysfunct 18:1007-1014
11. de Tayrac R, Chentouf S, Garreau H, Braud C, Guiraud I, Boudeville P, Vert M (2008) In vitro degradation and in vivo biocompatibility of poly(lactic acid) 94 mesh for soft tissue reinforcement in vaginal surgery. J Biomed Mater Res B Appl Biomater 85:529-536

12. Alponat A, Lakshminarasappa SR, Yavuz N, Goh PMY (1997) Prevention of adhesions by Seprefilm, an absorbable adhesion barrier: an incisional hernia model in rats. Am Surg 63:818-819

13. Zheng F, Lin Y, Verbeken E et al (2004) Host response after reconstruction of abdominal wall defects with porcine dermal collagen in a rat model. Am J Obstet Gynecol 191:1961-1970

14. Mathé ML, Lavigne JP, Oliva-Lauraire MC, Guiraud I, Marès P, de Tayrac R (2007) Comparison of different biomaterials for vaginal surgery using an in vivo model of meshes infection in rats. Gynécol Obstét Fertil 35:398-405

15. Hiltunen R, Nieminen K, Takala $T$ et al (2007) Low weight polypropylene mesh for anterior vaginal wall prolapse: a randomized controlled trial. Obstet Gynecol 110:455-462

16. Nguyen JN, Burchette RJ (2008) Outcome after anterior vaginal prolapse repair: a randomized controlled trial. Obstet Gynecol 111:891-898

17. Sivaslioglu AA, Unlubilgin E, Dolen I (2008) A randomized comparison of polypropylene mesh surgery with site specific surgery in the treatment of cystocele. Int Urogynecol J Pelvic Floor Dysfunct 19:467-471

18. Weyhe D, Hoffmann P, Belyaev O, Mros K, Muller C, Uhl W, Schmitz F (2007) The role of TGF-betal as a determinant of foreign body reaction to alloplastic materials in rat fibroblast cultures: comparison of different commercially available polypropylene meshes for hernia repair. Regul Pept 138(1):10-14, Epub 2006 Sep 12

19. Sergent F, Desilles N, Lacoume Y, Bunel C, Marie JP, Marpeau L (2009) Experimental biomechanical evaluation of polypropylene prostheses used in pelvic organ prolapse surgery. Int Urogynecol J 20:597-604

20. Judlin P (2002) Infections in gynecology. Masson, Paris, pp p6p7

21. Gristina AG (1987) Biomaterial-centered infection: microbial adhesion versus tissue integration. Science 237:1588-1595

22. Klinge U, Junge K, Spellerberg B, Piroth C, Klosterhalfen B, Schumpelick V (2002) Do multifilament alloplastic meshes increase the infection rate? Analysis of the polymeric surface, the bacteria adherence, and the in vivo consequences in a rat model. J Biomed Mater Res 63:765-771 